LBL-36501

$\mathrm{UC}-414$

\title{
Accelerator Waveform Synthesis and Longitudinal Beam Dynamics in a Small Induction Recirculator
}

\author{
T.J. Fessenden \\ Accelerator and Fusion Research Division \\ Lawrence Berkeley Laboratory \\ University of California \\ Berkeley, CA 94720 \\ D.P. Grote and W.M. Sharp \\ Lawrence Livermore National Laboratory \\ University of California \\ Livermore, CA 94550
}

April 1995

This work was supported in part by the Director, Office of Energy Research, Office of High Energy Physics, of the U.S. Department of Energy under Contract No. DE-AC03-76SF00098, and Lawrence Livermore National Laboratory under Contract No. W-7405-ENG-48. 


\section{DISCLAIMER}

This report was prepared as an account of work sponsored by an agency of the United States Government. Neither the United States Government nor any agency thereof, nor any of their employees, make any warranty, express or implied, or assumes any legal liability or responsibility for the accuracy, completeness, or usefulness of any information, apparatus, product, or process disclosed, or represents that its use would not infringe privately owned rights. Reference herein to any specific commercial product, process, or service by trade name, trademark, manufacturer, or otherwise does not necessarily constitute or imply its endorsement, recommendation, or favoring by the United States Government or any agency thereof. The views and opinions of authors expressed herein do not necessarily state or reflect those of the United States Government or any agency thereof. 


\section{DISCLAIMER}

Portions of this document may be illegible in electronic image products. Images are produced from the best available original document. 


\title{
ACCELERATOR WAVEFORM SYNTHESIS AND LONGITUDINAL BEAM DYNAMICS IN A SMALL INDUCTION RECIRCULATOR*
}

\author{
T.J. Fessenden, Lawrence Berkeley Laboratory \\ D.P. Grote and W.M. Sharp, Lawrence Livermore National Laboratory
}

\section{SUMMARY}

A recirculating induction accelerator requires accelerating waveforms that produce current amplification. and provide bunch length control throughout the acceleration process. Current amplification occurs because of both an increase in the beam velocity and a shortening of the length of the beam bunch. The pulsed acceleration and control waveforms seen by the beam change in time as the pulse duration shortens. For one acceleration cycle of the small recirculator, each accelerating gap is driven by a burst of 15 pulses. As the beam gains velocity, the time interval between pulses shortens from approximately 20 to $10 \mu \mathrm{sec}$. A zero-dimensional design code REC is used to develop the accelerator wave forms. An envelope/fluid code CIRCE and a 3-D particle code WARP3d are used to confirm the REC design and study the effects of errors. We find that acceleration errors can lead to space-charge waves launched at the bunch ends that strongly affect or even destroy the current pulse shape. The relation between the rate of longitudinal compression and the velocity of space charge waves is studied

\section{INTRODUCTION}

To serve as a heavy-ion accelerator/driver, a recirculator must operate at much larger currents than common in existing circular accelerators. In order to minimize collective particle effects, all acceleration must occur very rapidly--within a very few turns $(\approx 100)$. As in a linac, longitudinal bunch control must be supplied by the accelerating waveforms and current amplification during acceleration must be maintained. Much of the basic accelerator physics and beam manipulations of an induction recirculator can be examined in a "proof-ofprincipal" experiment. The companion paper of Friedman et al [1] at this conference describes a small experiment that is being assembled at the Lawrence Livermore National Laboratory to demonstrate many of the fundamental principles of this type of accelerator. This paper describes the development of the acceleration waveforms for this experiment along with physics studies of beam behavior under different acceleration scenarios.

The small recirculator design contains 20 full lattice periods each $72 \mathrm{~cm}$ long that form a ring approximately $4 \mathrm{~m}$ in diameter. Transverse focusing is provided by permanent magnet quadrupoles, and bending is supplied by electric dipoles. The reference design accelerates $2 \mathrm{~mA}$ of $\mathrm{K}^{+}$from an energy of $80 \mathrm{kV}$ to $320 \mathrm{kV}$ and a final current of $8 \mathrm{~mA}$. Acceleration occurs over 15 laps in a ${ }^{*}$ time

\footnotetext{
- Work performed under auspices of U.S. DoE by LLNL and LBL under contract W-7405-ENG-48 and DE-AC0376 SF00098.
}

period of approximately $230 \mu$ s. Current amplification results from both a doubling of the pulse speed and a halving of the bunch length during acceleration. Of the 40 half lattice period in the ring, four will be used for beam insertion and extraction and 36 will have gaps for applying the accelerating and longitudinal control waveforms (ears).

\section{COMPUTER CODES}

We have employed three different codes for developing the reference accelerating and control waveforms for the experiment. The first, REC, is a zero dimensional synthesis code that is useful for obtaining initial estimates of beam parameters and timing during acceleration. The REC code uses the acceleration procedure developed by C. Kim and L. Smith [2] to generate the proper waveforms to produce self-similar current amplification over the accelerator cycle. Transversely, the code assumes that the beam is completely dominated by space charge and always matched to the transport lattice. The effects of space charge on the longitudinal beam dynamics are ignored The code also calculates the electric dipole waveforms to keep the beam on axis and estimates the beam size at head, center, and tail during acceleration using the method developed by Lee $e t$ al.[3]. The waveforms and timing data from REC are used as input to the CIRCE and WARP codes.

CIRCE [4] is a fast-running macroscopic beamdynamics code that uses a truncated moment or "envelope" description of transverse dynamics, with equations for the radii and the centroid co-ordinates of the elliptical beam. Equations for longitudinal dynamics are obtained by treating the beam as a Lagrangian fluid. Proper terms are included to account for the effects of image forces, beam emittance, and space charge in the limit of paraxial motion, and the beam is focused and accelerated by a user-specified lattice of electric or magnetic dipoles and quadrupoles.

For these calculations the waveforms and timing data from REC are used to start the code and the longitudinal and transverse behavior of the beam in the recirculator is examined. In this paper, a particularly simple model of the beam space charge was used, in which the axial electric field averaged over the beam cross-section is proportional to the spatial derivative of the line-charge density multiplied by a logarithmic "geometry" factor.

WARP3d [5] is a three-dimensional particle-in-cell code simulation code that includes a framework for specifying a lattice of accelerating elements. The lattice elements can be modeled as finite-length, hard-edged elements where "residence corrections" are done to ensure that the correct impulses are given, or, for electric elements, can be modeled self-consistently by inclusion of 
the conductor geometry in the calculation of the fields. A warped coordinate system is used to model accelerator bends. The finite-length accelerating gaps can be time dependent. For these simulations, the hard edged quadrupole model was used and the electric dipole bending plates were included self-consistently.

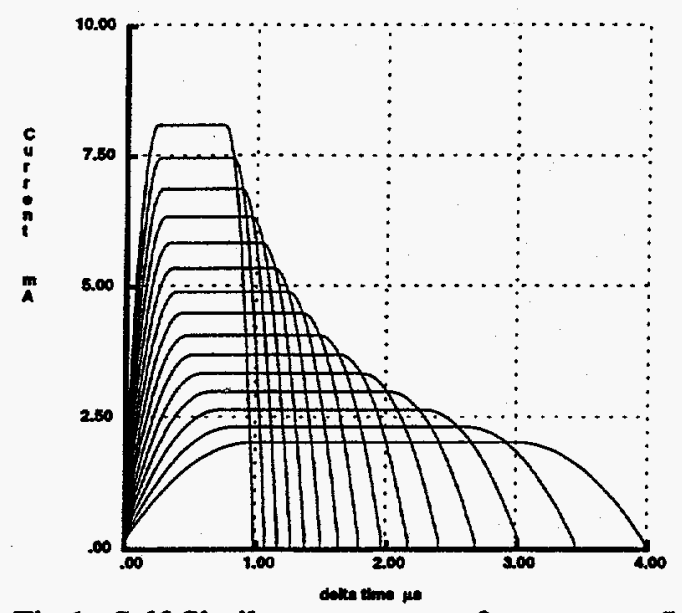

Fig 1. Self-Similar current waveforms at gap 5.

\section{RECIRCULATOR WAVEFORM DESIGN}

Figure 1 shows the anticipated self-similar current waveforms at gap 5 for each of the 15 turns of the beam generated by the REC code for the parameters of the small recirculator. Figure 2 shows the accelerating waveforms at gap 5 that are required to produce the current waveforms shown in Fig. 1. The length control waveforms (ears) are not shown. The first waveform applied to the first 8 gaps is essentially triangular. These accelerate the bunch tail more than the head and establish the proper velocity ramp for current amplification. The remaining 280 ideal waveforms (excluding ears) required when the beam is present in each gap are essentially rectangular.

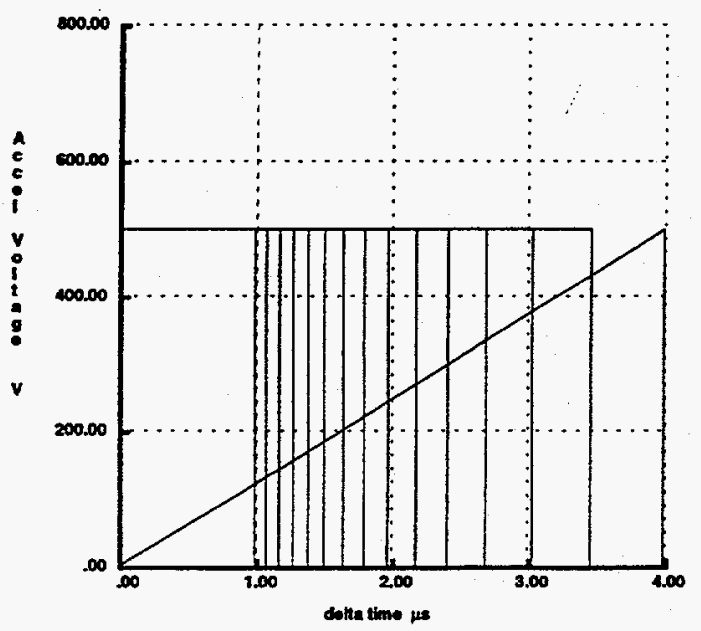

Fig 2. Ideal accelerator waveforms at gap 5 for self-similar current amplification

The waveforms that control the beam longitudinally are presented in Fig 3. These were calculated with CIRCE and are very close to those needed to confine the beam against its longitudinal space charge and axial temperature. Time is measured from beam center so that both the front and back "ears" can be seen.

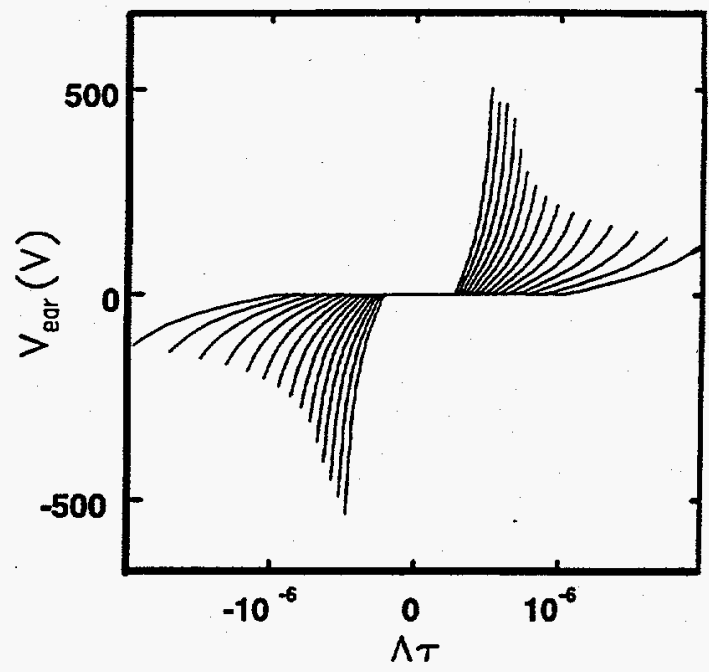

Fig. 3 Waveforms from CIRCE needed to provide longitudinal beam control for this design.

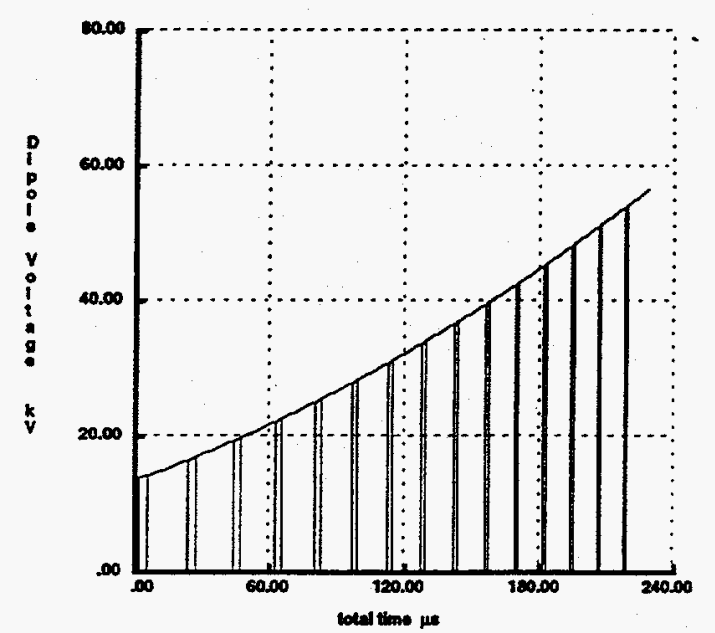

Fig. 4 Dipole bending voltages at gap 5 for the reference acceleration schedule

The voltage versus time that must be applied to each of the 40 bending dipoles of the recirculator for the acceleration schedule presented in this paper are shown in Fig. 4. The curve was calculated from the instantaneous energy of the beam center as it passes each bending dipole. This algorithm is only strictly correct for acceleration schedules that preserve the bunch length during acceleration. For the bunch-shortening schedule of this paper this simplification produces an error of $\leq 0.2 \%$. As a consequence all 40 bending quadrupoles may be powered from the same supply and no fine tweaking requiring 40 separate supplies is believed necessary. Also shown is the timing of the beam for the 15 passes of the beam past gap 5. The accelerator pulses must be carefully synchronized to the beam at each of the 36 active gaps. As the beam gains energy, the ideal accelerator pulse duration shortens as shown in Figs. 1, 2, and 3 and the interval between pulses decreases. 


\section{LONGITUDNAL DYNAMICS}

The CIRCE and WARP3d codes are used to study effects produced by errors in acceleration schedules. Fig 5. presents an example in which compression was attempted by only applying the "ear" control waveforms of Fig. 3 . That is, the initial triangular waveforms suggested by the REC code were not used Strong space charge waves are generated at the ends of the beams that propagate toward beam center and double when they collide at the center. As the waves separate longitudinal beam heating is observed and the code crashes before the beam completes 15 laps.

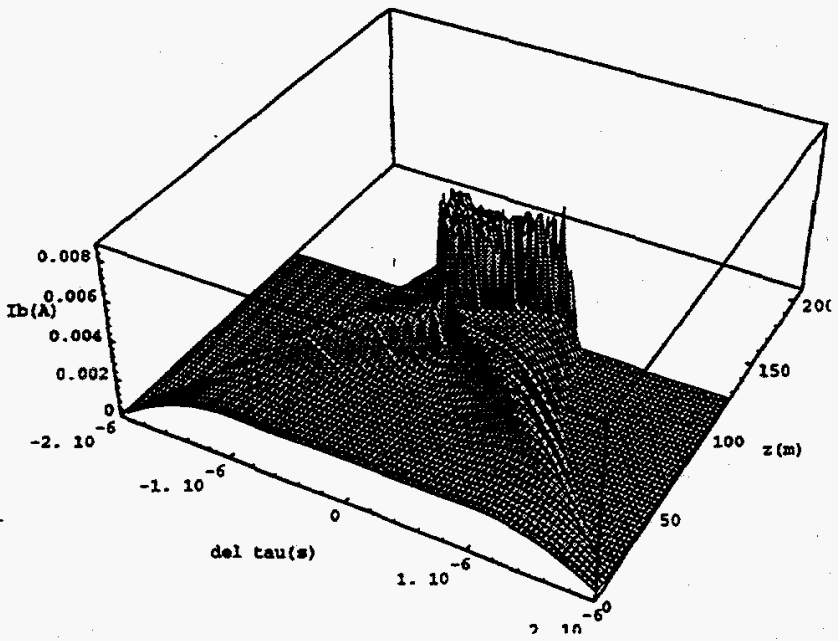

Fig. 5 Beam compression using only axial control waveforms (ears). The beam current is shown in the beam frame versus time and acceleration distance.

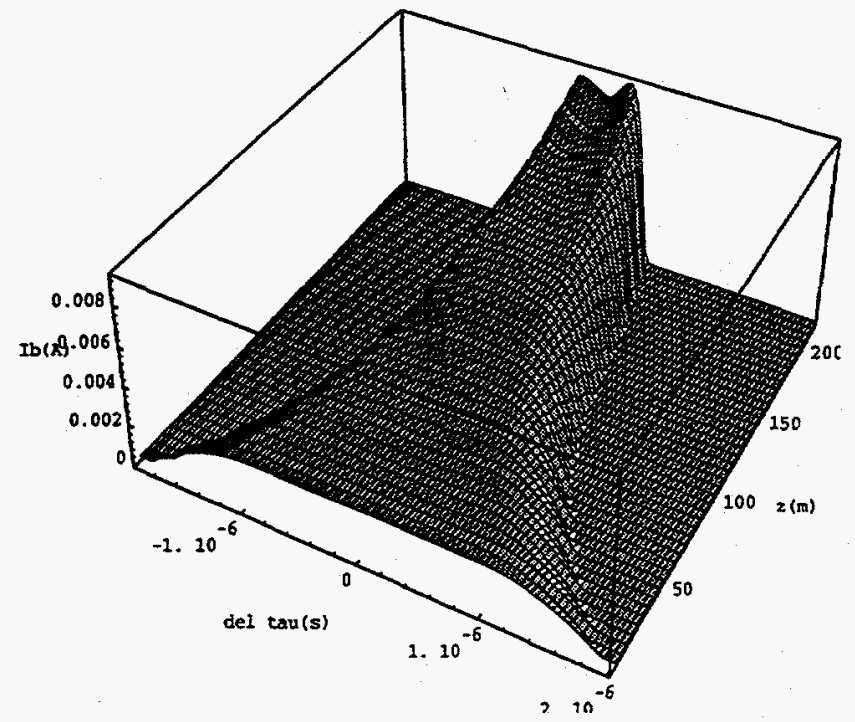

Fig. 6 CIRCE calculations of Beam compression using the compression schedule provided by REC.

A CIRCE calculation that incorporated all the waveforms and timing suggested by the REC code is shown in Fig. 6. This compression reasonably reproduces the self-similar current amplification of the REC code except over the last 50 meters of acceleration. WARP3d calculations using the REC waveforms and timing and the CIRCE "ears" of Fig. 3 is shown in Fig. 7. Much of the noise apparent in Fig. 7 is numerical.

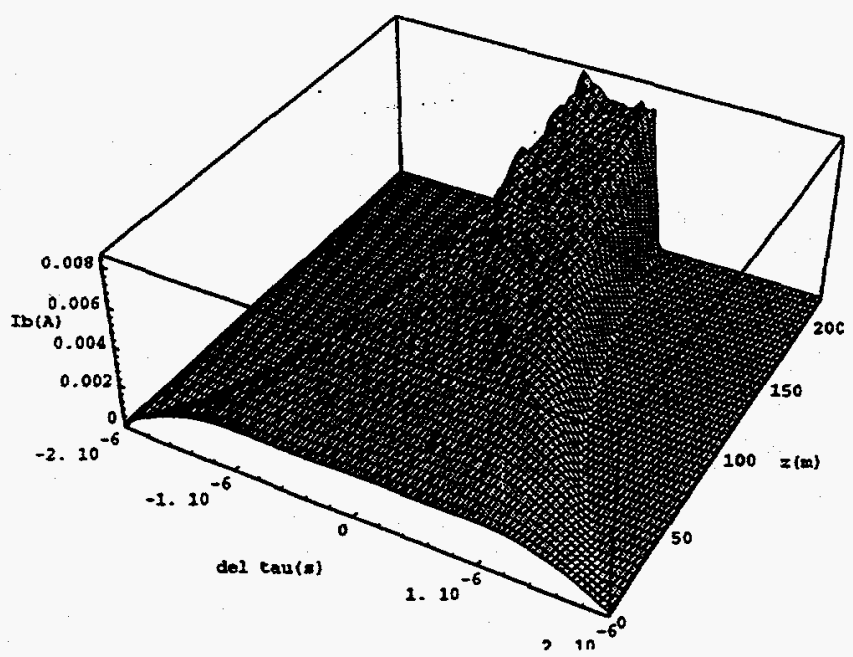

Fig. 7 WARP3d calculations of the same compression schedule as in Fig. 6.

\section{CONCLUSIONS}

The Kim-Smith self-similar current amplification procedure as embodied in the REC code provides an initial prescription and timing data for developing the acceleration waveforms needed for the small induction recirculator. Such a procedure is necessary for maintaining control of the current waveform over acceleration distances that are long compared with the length of the bunch. Moreover, bunch length compression must be effected by waveforms that act over the entire bunch length.

Results from the WARP3d and CIRCE codes are in good qualitative agreement. However, the algorithm used by the CIRCE code for generating the waveform control waveforms ("ears") used in these simulations was not quite adequate. As a consequence, neither code was able to preserve the self-similar current waveforms over the entire acceleration cycle. Further improvements in treating the effects of space charge at the bunch ends have been made and are reported in Ref. [6].

\section{REFERENCES}

[1] A. Friedman $e t$.al,. These proceedings.

[2] C.H. Kim and L. Smith, Part. Accel. 85, 101-103 (1986).

[3] E.P. Lee, T.J. Fessenden, and L.J. Laslett, IEEE Trans. Nuc. Sci. 32, 2589 (1985).

[4] W. M. Sharp, J. J. Barnard, D. P. Grote, S. M. Lund, and S. S. Yu, AIP Press, 297, R. D. Ryne, ed., pp. 540-548 (1994).

[5] A. Friedman, D. P. Grote, and I. Haber, Phys. Fluids B, 4, p. 2203 (1992).

[6]W. M. Sharp, These proceedings. 